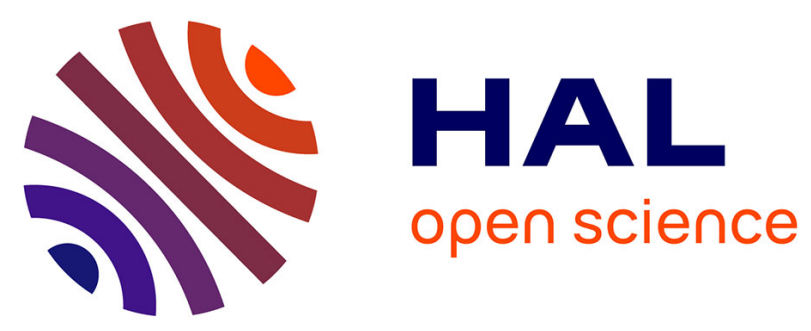

\title{
La contribution des écarts de rémunération entre les femmes et les hommes à l'inégalité des rémunérations dans la fonction publique: une approche par la décomposition des inégalités
}

Frédéric Chantreuil, Frédéric Gavrel, Jean-Pascal Guironnet, Isabelle Lebon

\section{To cite this version:}

Frédéric Chantreuil, Frédéric Gavrel, Jean-Pascal Guironnet, Isabelle Lebon. La contribution des écarts de rémunération entre les femmes et les hommes à l'inégalité des rémunérations dans la fonction publique: une approche par la décomposition des inégalités. Economie et Statistique / Economics and Statistics, 2016, 488-489 (1), pp.152-168. 10.3406/estat.2016.10716 . halshs-01377617

\section{HAL Id: halshs-01377617 \\ https://shs.hal.science/halshs-01377617}

Submitted on 7 Oct 2016

HAL is a multi-disciplinary open access archive for the deposit and dissemination of scientific research documents, whether they are published or not. The documents may come from teaching and research institutions in France or abroad, or from public or private research centers.
L'archive ouverte pluridisciplinaire HAL, est destinée au dépôt et à la diffusion de documents scientifiques de niveau recherche, publiés ou non, émanant des établissements d'enseignement et de recherche français ou étrangers, des laboratoires publics ou privés. 


\title{
La contribution des écarts de rémunération entre les femmes et les hommes à l'inégalité des rémunérations dans la fonction publique : une approche par la décomposition des inégalités
}

\author{
Frédéric Chantreuil *, Frédéric Gavrel *, Jean-Pascal Guironnet * \\ et Isabelle Lebon *
}

Le Système d'information sur les agents des services publics retrace la rémunération annuelle de chacun des salariés de la fonction publique. Du fait de son exhaustivité, il constitue une base de données particulièrement intéressante pour appréhender les écarts de rémunération entre les femmes et les hommes. Cet article s'intéresse à la contribution de cet écart à l'inégalité des rémunérations entre l'ensemble des salariés de ce secteur. On considère la rémunération nette totale reçue par chaque agent comme la somme de plusieurs éléments, l'un propre à son âge pour capter son avancement dans la carrière, un deuxième déterminé par son sexe et un dernier associé à d'autres caractéristiques ; on évalue alors la contribution du genre aux inégalités de rémunération, mesurées par l'indice de Gini, avec une décomposition inspirée de la valeur de Shapley.

Cette décomposition attribue aux écarts de rémunération entre les hommes et les femmes une contribution de $10 \%$ pour la catégorie $\mathrm{A}, 10,6 \%$ pour la catégorie $\mathrm{B}$ et $11,7 \%$ pour la catégorie C. D'importantes disparités apparaissent entre les différents versants de la fonction publique. Dans la fonction publique hospitalière, la contribution du genre est très faible hors catégorie $\mathrm{A}$. Au contraire, dans la fonction publique d'État, toutes les catégories affichent une contribution du genre supérieure à $10 \%$, particulièrement dans

Rappel :

Les jugements et opinions exprimés par les auteurs n'engagent qu'eux mêmes, et non les institutions auxquelles ils appartiennent, ni a fortiori l'Insee. les administrations centrales. Dans la fonction publique territoriale, la part des inégalités de rémunération attribuable aux différences de rémunération femmes-hommes est plus importante dans les catégories $\mathrm{A}$ et $\mathrm{C}$ que dans la catégorie $\mathrm{B}$, et dans les autres établissements publics locaux que dans les collectivités territoriales.

Codes JEL : C71, D63, J16.

Mots clés : inégalité, décomposition par facteurs, Shapley, genre.

Les auteurs souhaitent remercier Mathieu Bunel pour sa participation active au contrat dont est issu ce travail (Bunel et al., 2014), Alpha Oumar Diop pour le traitement statistique de la base de données SIASP 2010, et les deux rapporteurs anonymes pour des remarques et suggestions qui ont largement contribué à l'amélioration de cet article.

Cette étude a bénéficié du financement de la Direction générale de l'Administration et de la fonction publique et du Défenseur des Droits (convention $n^{\circ}$ 2200730964).

Ce travail a bénéficié d'une aide de L'État gérée par l'Agence nationale de la Recherche au titre du programme Investissements d'avenir portant la référence ANR-10-EQPX-17 (Centre d'accès sécurisé aux données du Genes - CASD). 
T outes les études, et notamment les rapports Guégot (2011) et DGAFP (2012), dressent un constat identique à propos des rémunérations dans la fonction publique : les femmes y sont en moyenne moins bien rémunérées que les hommes. Ce résultat général se vérifie également lorsque chaque versant est envisagé séparément. Le rapport de la DGAFP (2012) met ainsi en évidence pour l'année 2010 des écarts de traitement de $10,6 \%$ dans la fonction publique territoriale et de 13,9\% dans la fonction publique d'État. Les déterminants des écarts de salaire entre les femmes et les hommes ont fait l'objet de très nombreux travaux et sont maintenant bien documentés sur le plan empirique; ils combinent toujours des différences de temps de travail, d'expérience accumulée, d'emplois occupés, et relèvent aussi parfois de ces « barrières invisibles » qui bloquent les femmes dans leur progression de carrière. Ces mêmes facteurs existent, à des degrés divers, dans le secteur privé comme dans la fonction publique (voir les explorations récentes présentées par Duvivier et al. et Fremigacci et al. dans ce numéro). À la différence de ces travaux, notre objectif n'est pas d'expliquer les écarts de rémunérations entre les femmes et les hommes, mais de mesurer leur contribution à l'inégalité totale des rémunérations observée dans la fonction publique.

Pour établir le niveau des inégalités et mesurer la part éventuellement attribuable aux différences de rémunération entre hommes et femmes, nous mobilisons les données du Système d'information sur les agents des services publics (SIASP) pour l'année 2010. Cette base exhaustive regroupe des informations sur les rémunérations, le sexe, l'âge, le nombre d'heures de travail effectif au cours de l'année, le statut, la catégorie du poste et l'établissement de rattachement de l'ensemble des salariés des trois versants de la fonction publique : fonction publique d'État (FPE), Territoriale (FPT) et Hospitalière (FPH).

La présente étude s'inscrit donc dans une littérature qui s'intéresse à la décomposition des indices d'inégalité. La décomposition des inégalités de revenu vise usuellement à déterminer quelle proportion de ces inégalités est attribuable à une source de revenu ou à une sous-population particulière. Par exemple, on peut chercher à déterminer quelle part des inégalités de revenu des ménages est attribuable à la dispersion de chacune des composantes de ce revenu (les revenus du travail, les revenus du capital, etc.) ou, alternativement, comment la dispersion des revenus à l'intérieur de tel ou tel sous-groupe d'individus contribue à expliquer les inégalités observées sur la population entière. Une décomposition par sous-population peut ainsi permettre de mesurer quelles proportions des inégalités sont expliquées par les disparités de revenus des hommes entre eux et des femmes entre elles (voir par exemple Koubi et al., 2005). En revanche, la décomposition par sous-population ne dit rien de la contribution aux inégalités des disparités de revenu qui pourraient exister entre les hommes et les femmes. Pour traiter spécifiquement de cette question, une nouvelle approche de la décomposition des inégalités doit être envisagée.

Inspirée de Shapley (1953), la méthode de décomposition proposée ici est une adaptation des travaux de Chantreuil et Trannoy $(2011,2013)$ permettant d'évaluer non plus la contribution marginale d'une source de rémunération ou d'une sous-population à l'inégalité globale, mais celle qui peut être liée à une caractéristique des individus, ici le fait d'être un homme ou une femme. Une contribution positive aux inégalités de ce facteur de « genre » attesterait d'un décalage entre les distributions de rémunérations des femmes et des hommes, décalage d'autant plus grand que la contribution serait élevée. Cette méthodologie, mise en œuvre tour à tour dans les différents secteurs de la fonction publique, permet d'appréhender dans quelles catégories et quels types d'administration les écarts de rémunérations entre les hommes et les femmes contribuent le plus à l'inégalité.

\section{Une décomposition inspirée de la « valeur de Shapley»}

A priori, dans le secteur public, la rémunération d'un agent dépend principalement de sa catégorie hiérarchique, de son ancienneté, et de son temps de travail; elle peut également être différente selon le versant de rattachement et le type d'établissement employeur. La source SIASP ne donne toutefois pas d'information sur l'année d'entrée des agents dans la fonction publique, ni sur leurs éventuelles interruptions de carrière; on utilise donc l'âge des agents comme proxy de l'ancienneté, comme il est courant de le faire dans les travaux menés sur cette base de données. Les rémunérations des agents sont ramenées à leur « équivalent temps complet » (EQTP) en rapportant la durée annuelle travaillée à la durée 
théorique d'un temps plein annuel, à savoir 1820 heures (cf. encadré 1 pour une présentation plus complète des données). Ce calcul induit une légère surestimation de la rémunération en EQTP des agents travaillant pour des quotités de $80 \%$ et de $90 \%$ de temps, qui sont rémunérées un peu plus que proportionnellement. Parce qu'elles sont les plus concernées par ces durées, c'est la rémunération moyenne en EQTP des femmes qui est le plus tirée à la hausse par le passage à l'équivalent temps plein.

L'inégalité des rémunérations EQTP va alors être décomposée en tenant compte de l'âge (comme proxy de l'avancement dans la carrière) et du sexe des agents; les autres caractéristiques telles que la catégorie, le versant et l'établissement d'appartenance seront prises en compte par des décompositions réalisées séparément sur chacun des segments de la fonction publique. C'est l'indice de Gini qui, étant donné son caractère standard, a été retenu pour l'évaluation de l'inégalité totale des rémunérations et qui est décomposé. Le niveau de cet indice dépend de la distribution, plus ou moins dispersée, des rémunérations et varie entre 0 (situation où toutes les rémunérations seraient identiques) et 1 (situation où toutes les rémunérations sauf une seraient nulles).

La décomposition mise en œuvre relève d'une méthodologie originale inspirée de la valeur de Shapley (Chantreuil et Lebon, 2015). Dans un premier temps, on considère que la rémunération résulte de trois sources : l'une spécifique à l'âge de l'agent, l'une liée au sexe de l'agent et l'une propre aux autres caractéristiques, inobservées, de l'agent. La détermination de ces trois distributions permet ensuite de calculer la contribution de chacun de ces trois éléments à l'inégalité totale des rémunérations ; autrement dit, l'inégalité mesurée sur la distribution des rémunérations totales, telle qu'elle

Encadré 1

\section{SOURCE, CHAMP ET DÉFINITIONS}

L'étude porte sur les salariés de la fonction publique présents sur le territoire national et dans les DOM, actifs au 31 décembre 2010, soit, dans SIASP 2010 une population de 5765960 agents.

Nous excluons de cet ensemble:

(1) Les observations présentant des informations manquantes: grade, catégorie ou PCS (Professions et catégories socioprofessionnelles) non renseignés; statut de contrat inconnu ; nombre d'heures de travail non précisé ;

(2) Les observations pour lesquelles des valeurs sont aberrantes: salaires nets et/ou bruts négatifs ou nuls; des salaires nets annualisés supérieurs à 200000 euros pour les catégories B et C, et supérieurs à 250000 euros pour des catégories $A$ touchant moins de 100000 euros de primes; des primes négatives; des quotités nulles; des salaires bruts moins primes négatifs ou inférieurs au traitement minimum brut de la fonction publique pour les catégories $A$ et $B$ à temps plein ; des salaires nets annualisés inférieurs au traitement minimum net de la fonction publique pour les catégories A et B ; des salaires nets annualisés inférieurs au SMIC net pour les catégories $C$; des salaires bruts inférieurs au SMIC brut pour les agents catégories $\mathrm{C}$ à temps plein ; des parts de primes supérieures à $75 \%$;

(3) Les observations présentant des caractéristiques qui rendent impossible un traitement homogène des données: postes identifiés comme inactifs ou annexes; des postes spécifiés comme étant hors fonction publique; quelques personnels sous statut militaire (sapeur pompier de Marseille...), les militaires ayant été exclus de la base fournie ; des élus ; des élèves de la FPH (autres que les internes) ; des travailleurs saisonniers ou occasionnels, des travailleurs payés à l'acte, à la tâche ou des rémunérations accessoires ; des emplois aidés et des apprentis ; des personnes en FPA ou service civique.

Par ailleurs, seuls les agents âgés d'au moins 18 et de 65 ans au plus et, pour des raisons de standardisation, ayant travaillé en 2010 un nombre d'heures au moins égal à un mi-temps sur un mois ont été retenus dans l'étude.

Le champ tel que défini ci-dessus comporte 4637701 salariés (titulaires et non-titulaires) de la fonction publique. Les non-titulaires représentent $15,4 \%$ de ces salariés ; ils sont présents dans tous les versants, à raison de 13,8 \% dans la FPE, de 17,4\% dans la FPH et de $15,8 \%$ dans la FPT, et dans toutes les catégories (15,8\% pour la catégorie A, 14,3\% pour la catégorie B et 15,5\% pour la catégorie C).

$19,1 \%$ des salariés de la fonction publique sont à temps partiel et $5,9 \%$ ont un temps de travail supérieur à un temps plein. Pour rendre comparables les rémunérations de l'ensemble des agents, toutes sont ramenées à un équivalent temps plein, soit 1820 heures annuelles. Les rémunérations prises en compte dans l'étude comprennent les salaires et les primes des agents, les heures supplémentaires ou complémentaires, et éventuellement le supplément familial de traitement, sachant que les données disponibles ne permettent pas de distinguer ces revenus annexes des primes elles-mêmes. Elles sont nettes des cotisations sociales. 
est observée, est décomposée en fonction des trois distributions qui sont formellement traitées comme trois sources de revenu (Chantreuil et Trannoy, 2011, 2013). Plus précisément, la construction des trois distributions est réalisée de la façon suivante :

Le montant spécifique à l'âge est commun à tous les agents du même âge et supposé égal à la rémunération moyenne de tous les agents de la sous-population nés la même année. Pour les agents d'âge $a$, la variable ainsi construite s'écrit : $\bar{x}(a)$.

Le terme spécifique au sexe de l'agent, noté $g$, représente l'éventuel écart de rémunération entre les femmes et les hommes. Il est calculé comme la différence entre la rémunération moyenne des femmes $(f)$, respectivement des hommes $(m)$, d'âge $a$ et la rémunération moyenne de l'ensemble des agents de cet âge. Cette différence prend la forme suivante: $[\bar{x}(a, g)-\bar{x}(a)]$ avec $g=(f, m)$. Dans la suite de l'article, nous appellerons cette source le «facteur genre», et elle sera évidemment au cœur de l'étude de la contribution des disparités entre femmes et hommes à l'inégalité totale des rémunérations.

Enfin, la partie spécifique à l'agent $i$, qui est liée à des caractéristiques autres que son âge ou son sexe, correspond à la différence, entre la rémunération de cet agent, $x(i)$ et la rémunération moyenne des agents du même âge et du même sexe. Pour un agent $i$ d'âge $a$ et de sexe $g$, elle est égale à : $[x(i)-\bar{x}(a, g)]$,
Avec ces notations, la rémunération d'une femme $i$ d'âge $a$ prend donc la forme de la somme suivante :

$$
x(a, f, i)=\bar{x}(a)+[\bar{x}(a, f)-\bar{x}(a)]+[x(i)-\bar{x}(a, f)],
$$

Le premier terme (la rémunération moyenne des agents du même âge qu'elle) prend toujours une valeur strictement positive.

Le second terme (la différence entre la rémunération moyenne des femmes et celle de tous les agents du même âge) peut être positif ou négatif selon que les femmes d'âge $a$ gagnent en moyenne plus ou moins que l'ensemble des agents d'âge $a$. Si un décalage existe entre les rémunérations des hommes et des femmes, il y aura donc une contribution positive du « facteur genre » à l'inégalité.

Le dernier terme (la différence entre la rémunération de cette femme $i$ et la rémunération moyenne des femmes de son âge) peut être positif ou négatif selon que cette salariée gagne plus ou moins que la moyenne des femmes de son âge.

C'est sur cette base qu'une décomposition à la Shapley par source de revenu est ensuite mise en œuvre. Le principe consiste à mesurer la différence entre l'inégalité de la distribution de l'ensemble des rémunérations telle qu'elle est observée et l'inégalité de la distribution des rémunérations que l'on observerait si l'une ou l'autre des sources disparaissait (voir l'encadré 2 pour une présentation formelle de la décomposition à la Shapley).

Encadré 2

\section{MESURER LA CONTRIBUTION DU GENRE AUX INÉGALITÉS, UNE DÉCOMPOSITION INSPIRÉE DE LA VALEUR DE SHAPLEY}

En utilisant la théorie des jeux coopératifs, il est possible de définir une méthode de décomposition de la mesure des inégalités qui s'applique aussi bien à la décomposition par sous-population qu'à la décomposition par source de revenu: la décomposition de Shapley (Chantreuil et Trannoy, 2011, 2013 et Chantreuil et Lebon, 2015). Dans ce cadre, la contribution marginale directe d'une source de revenu à l'inégalité totale est définie comme étant la différence entre l'inégalité totale et l'inégalité de la distribution de revenu si cette source disparaissait. L'avantage de cette méthode par rapport à celles usuellement proposées dans la littérature pour appréhender ce type d'inégalité est double. D'une part, elle permet de tenir compte des décalages qui peuvent exister entre les rémunérations des hommes et des femmes, non pas à un niveau particulier comme la médiane ou la moyenne, mais sur la totalité de la distribution. D'autre part, elle s'applique quel que soit le type d'indicateur utilisé pour mesurer les inégalités. L'inconvénient majeur de la décomposition de Shapley est d'être sensible au nombre de sources de revenu composant le revenu global. Si on procède à plusieurs décompositions, elle doit donc être mise en œuvre avec un nombre de sources de revenu constant, afin que les résultats des différentes décompositions soient comparables.

Formellement, en écrivant la rémunération d'un agent comme la somme des trois composantes "âge ", «genre» et «reste» incluant les autres caractéristiques, nous transposons la décomposition par 
Encadré 2 (suite)

source pour déterminer la contribution du différentiel de rémunération entre femmes et hommes (le facteur " genre ") à l'inégalité totale des rémunérations. Si la source " genre » prend une valeur strictement positive, cela reflète l'existence d'un écart systématique, en défaveur des femmes, entre les rémunérations reçues par les hommes et celles reçues par les femmes.
Pour s'en convaincre prenons l'exemple des deux distributions salariales présentées dans le tableau $A$. Chacune des deux distributions a les caractéristiques suivantes: elle regroupe 40 personnes réparties entre 5 tranches d'âge; chaque tranche se compose de 4 hommes et de 4 femmes dont les salaires progressent régulièrement dans le temps.

Tableau A

Exemple de deux distributions salariales type

\begin{tabular}{|c|c|c|c|c|}
\hline & \multicolumn{2}{|c|}{ Distribution initiale } & \multicolumn{2}{|c|}{ Distribution $\mathrm{H}(+50) / \mathrm{F}(-50)$} \\
\hline & Salaires $\mathrm{H}$ & Salaires $\mathrm{F}$ & Salaires $\mathrm{H}$ & Salaires F \\
\hline Âge $=1$ & $\begin{array}{l}300 ; 400 ; \\
600 ; 700\end{array}$ & $\begin{array}{l}400 ; 450 ; \\
500 ; 650\end{array}$ & $\begin{array}{l}350 ; 450 ; \\
650 ; 750\end{array}$ & $\begin{array}{l}350 ; 400 ; \\
450 ; 600\end{array}$ \\
\hline Âge $=2$ & $\begin{array}{l}350 ; 450 ; \\
650 ; 750\end{array}$ & $\begin{array}{l}450 ; 500 ; \\
550 ; 700\end{array}$ & $\begin{array}{l}400 ; 500 ; \\
700 ; 800\end{array}$ & $\begin{array}{l}400 ; 450 ; \\
500 ; 650\end{array}$ \\
\hline Âge $=3$ & $\begin{array}{l}400 ; 500 ; \\
700 ; 800\end{array}$ & $\begin{array}{l}500 ; 550 ; \\
600 ; 750\end{array}$ & $\begin{array}{l}450 ; 550 ; \\
750 ; 850\end{array}$ & $\begin{array}{l}450 ; 500 ; \\
550 ; 700\end{array}$ \\
\hline Âge $=4$ & $\begin{array}{l}450 ; 550 ; \\
750 ; 850\end{array}$ & $\begin{array}{l}550 ; 600 ; \\
650 ; 800\end{array}$ & $\begin{array}{l}500 ; 600 ; \\
800 ; 900\end{array}$ & $\begin{array}{l}500 ; 550 ; \\
600 ; 750\end{array}$ \\
\hline Âge $=5$ & $\begin{array}{l}500 ; 600 ; \\
800 ; 900\end{array}$ & $\begin{array}{l}600 ; 650 ; \\
700 ; 850\end{array}$ & $\begin{array}{l}550 ; 650 ; \\
850 ; 950\end{array}$ & $\begin{array}{l}550 ; 600 ; \\
650 ; 800\end{array}$ \\
\hline
\end{tabular}

Dans la première distribution, dite distribution initiale, les hommes et les femmes ne reçoivent pas exactement les mêmes rémunérations, mais des rémunérations qui sont en moyenne identiques pour chacune des 5 tranches d'âge considérées. Dans ces conditions, la décomposition montre que la part de l'inégalité totale (mesurée par l'indice de Gini) qui peut être attribuée au facteur genre est nulle (cf. tableau B). En revanche, si, pour un salaire moyen identique à tous les âges à ceux de l'exemple précédent, les salaires de tous les hommes sont augmentés d'un montant égal à 50 alors que ceux de toutes les femmes sont diminués du même montant (distribution $\mathrm{H}(+50) / \mathrm{F}(-50)$ du tableau $\mathrm{A})$, on voit que la proportion de l'inégalité globale attribuable au facteur genre atteint $13,2 \%$. La méthode permet donc bien d'identifier le décalage qui pourrait exister entre les distributions des rémunérations des hommes et des femmes, comme d'en comparer l'importance dans différentes sous-populations.

\section{Tableau B}

Indice de Gini et contributions des trois sources

\begin{tabular}{|l|c|c|c|}
\hline & Distribution initiale & Distribution $\mathrm{H}(+50) / \mathrm{F}(-50)$ & Distribution $\mathrm{H}(+100) / \mathrm{F}(-100)$ \\
\hline Indice de Gini & 0,141 & 0,147 & 0,168 \\
Âge (\%) & 30,2 & 26,2 & 21,7 \\
Genre (\%) & 0,0 & 13,2 & 29,7 \\
Reste (\%) & 69,8 & 60,6 & 48,5 \\
\hline
\end{tabular}

Dans notre exemple, une sous-population dans laquelle les différences salariales entre les hommes et les femmes correspondraient à une augmentation de 100 des salaires des hommes et une baisse de 100 des salaires des femmes par rapport à la distribution initiale, afficherait en effet une contribution de $29,7 \%$ du facteur genre. La comparaison du niveau de cette contribution permet donc d'appréhender l'écart plus ou moins élevé qui existe entre les rémunérations des hommes et des femmes, et ce même lorsque cet écart n'est pas régulier sur l'ensemble de la distribution contrairement à ce qui apparaît dans cet exemple simple.

Pour établir la signification exacte de la contribution du facteur genre ainsi mesurée, il faut détailler la décomposition de l'indice de Gini de la façon suivante :

$$
\text { Gini }=S h_{a}+S h_{g}+S h_{R}
$$

Où $S h_{a}$ représente la contribution totale de l'âge, $S h_{g}$ celle du genre, et $S h_{R}$ celle du reste. 
La valeur de Shapley de chaque facteur se calcule alors de la façon suivante, sachant que l'on note $G(., .,$.$) le Gini de la distribution des revenus obtenus$ en sommant les termes indiqués dans la parenthèse :

$$
\begin{aligned}
S h_{a}= & \frac{1}{3} G\left(a, \mu_{g}, \mu_{R}\right)+\frac{1}{6}\left[G\left(a, g, \mu_{R}\right)-G\left(\mu_{a}, g, \mu_{R}\right)\right]+ \\
& \frac{1}{6}\left[G\left(a, \mu_{g}, R\right)-G\left(\mu_{a}, \mu_{g}, R\right)\right]+ \\
& \frac{1}{3}\left[G(a, g, R)-G\left(\mu_{a}, g, R\right)\right] \\
S h_{g}= & \frac{1}{3} G\left(\mu_{a}, g, \mu_{R}\right)+\frac{1}{6}\left[G\left(a, g, \mu_{R}\right)-G\left(a, \mu_{g}, \mu_{R}\right)\right]+ \\
& \frac{1}{6}\left[G\left(\mu_{a}, g, R\right)-G\left(\mu_{a}, \mu_{g}, R\right)\right]+ \\
& \frac{1}{3}\left[G(a, g, R)-G\left(a, \mu_{g}, R\right)\right] \\
S h_{R}= & \frac{1}{3} G\left(\mu_{a}, \mu_{g}, R\right)+\frac{1}{6}\left[G\left(a, \mu_{g}, R\right)-G\left(\mu_{a}, \mu_{g}, R\right)\right]+ \\
& \frac{1}{6}\left[G\left(\mu_{a}, g, R\right)-G\left(\mu_{a}, g, \mu_{R}\right)\right]+ \\
& \frac{1}{3}\left[G(a, g, R)-G\left(a, g, \mu_{R}\right)\right]
\end{aligned}
$$

Les poids utilisés dans la formule de la valeur de Shapley correspondent à la probabilité d'occurrence de chaque coalition de facteurs, sachant qu'avec 3 facteurs, il existe 3 types de coalitions : à un facteur, deux facteurs et trois facteurs (voir aussi Eidelman, 2012, pour une présentation complète de l'interprétation de la formule de la valeur de Shapley). Prenons l'exemple de la contribution totale du genre, $S h_{g}$, pour interpréter les termes et expliciter les pondérations utilisées. Cette contribution totale du genre est égale à la somme pondérée de plusieurs éléments :

(1) le Gini d'une distribution de revenu dans laquelle la variable âge et la variable reste prendraient leur valeur moyenne sur l'ensemble de la distribution (respectivement $\mu_{a}$ et $\mu_{R}$ ) et le genre ses valeurs réelles $G\left(\mu_{a}, g, \mu_{R}\right)$; cela correspond à une coalition ne comportant qu'un seul facteur, le genre, chaque facteur ayant une probabilité équivalente d'être tiré, la pondération est égale à $1 / 3$;

(2) la différence entre le Gini d'une distribution dans laquelle les attributs âge et genre prendraient leurs valeurs réelles et les autres caractéristiques individuelles leur valeur moyenne et le Gini d'une distribution où le genre serait aussi ramené à sa valeur moyenne $\left[G\left(a, g, \mu_{R}\right)-G\left(a, \mu_{g}, \mu_{R}\right)\right]$; cela correspond à une coalition comportant deux facteurs, tout d'abord le genre tiré avec une probabilité de $1 / 3$ puis l'âge tiré avec une probabilité de $1 / 2$ entre les deux facteurs restant, soit une pondération de $1 / 3 \times 1 / 2=1 / 6$;

(3) la différence entre le Gini d'une distribution dans laquelle le genre et le reste prendraient leurs valeurs réelles et l'âge sa valeur moyenne et le Gini d'une distribution où le genre serait aussi ramené à sa valeur moyenne $\left[G\left(\mu_{a}, g, R\right)-G\left(\mu_{a}, \mu_{g}, R\right)\right]$; la pondération se calcule de façon équivalente à celle du terme (2) ;

(4) la différence entre le Gini de la distribution effective des revenus et le Gini de la distribution lorsque le genre est ramené à sa valeur moyenne $\left[G(a, g, R)-G\left(a, \mu_{g}, R\right)\right]$; il s'agit alors d'une coalition à trois facteurs qui peut être obtenue après l'ajout du facteur restant à partir de n'importe laquelle des deux coalition de deux facteurs, chacune de ces coalition de deux facteurs avait une probabilité $1 / 6$ de survenir ; la pondération de la coalition de trois facteurs se déduit de la somme de leur probabilité d'occurrence, soit $1 / 6+1 / 6=1 / 3$.

La première partie de cette somme (1) peut être considérée comme l'effet direct du facteur genre, alors que les différences (2), (3) et (4) reflètent ses effets croisés avec l'âge et le reste. La contribution relative (le \%) associée à chacun des facteurs (voir tableau B) se calcule comme le ratio de la contribution totale de ce facteur au Gini total de la distribution. Par exemple dans le cas du genre: $S h_{g} /$ Gini .

\section{La contribution du « facteur genre » à l'inégalité : pas seulement un effet de la répartition des emplois par catégorie}

La méthode de décomposition est mise en œuvre pour déterminer la part attribuable au genre dans l'inégalité des rémunérations mesurée au niveau de la fonction publique en général ou d'une sous-population en particulier.

Notre analyse porte sur les salariés de la fonction publique pour lesquels l'ensemble des informations nécessaires sont renseignées et dont le poste constitue un emploi principal, soit 4637701 observations (voir l'encadré 1 pour plus de précisions sur le champ de l'étude). $40,1 \%$ de ces salariés appartiennent à la FPE, $23,3 \%$ à la FPH et $36,6 \%$ à la FPT. $32,4 \%$ appartiennent à la catégorie $\mathrm{A}$ et peuvent être assimilés à des cadres; ces emplois sont particulièrement concentrés dans la FPE, car la quasi-totalité des enseignants du primaire et du secondaire relève de cette catégorie. Quant aux emplois des catégories B et C lesquels, dans la classification par PCS peuvent être rapprochés respectivement des professions intermédiaires et des ouvriers/employés, ils représentent $20,1 \%$ et $47,5 \%$ des agents de notre champ. Les postes de la catégorie $\mathrm{C}$ sont en nombre particulièrement élevés dans la FPH et plus encore 
dans la FPT. L'âge moyen des agents est de 44 ans et demi pour les hommes et 42 ans pour les femmes. La grande majorité d'entre eux sont titulaires, mais les non-titulaires représentent néanmoins 15,4\% des salariés considérés. Ces non-titulaires, présents dans tous les versants et toutes les catégories, sont plus jeunes en moyenne que les titulaires.

La caractéristique du secteur public est un taux de féminisation très élevé, puisque $64,1 \%$ des salariés de notre base sont des femmes. Les femmes sont majoritaires dans tous les segments de la fonction publique à l'exclusion des emplois à responsabilités et des emplois de direction qui sont plus souvent occupés par des hommes. Les femmes représentent 85,6 \% des $19,1 \%$ d'agents à temps partiel. Le temps partiel concernant principalement les catégories B et $\mathrm{C}$, il est plus fréquent dans les secteurs qui comptent le plus de postes dans ces catégories, à savoir la FPH et la FPT.

Avec la méthodologie mise en œuvre, une valeur élevée de la contribution du facteur genre va de pair avec des écarts de rémunération entre les femmes et les hommes conséquents; il convient donc de commencer par présenter l'inégalité des rémunérations et leur décomposition pour mettre en évidence un éventuel décalage.

$\mathrm{Au}$ niveau de la fonction publique dans son ensemble, l'indice de Gini prend une valeur égale à 0,221 sur la rémunération totale (cf. tableau 1). Les salariés de la fonction publique étant ainsi considérés dans leur globalité, sans distinction quant à l'administration dans laquelle ils travaillent ou à la catégorie à laquelle ils appartiennent, l'âge (c'est-à-dire l'avancement dans la carrière) contribue pour $17,2 \%$ à l'inégalité de rémunérations.

L'impact de l'âge étant pris en compte, la décomposition fait apparaître une contribution du « facteur genre » à l'inégalité des rémunérations de près de $9 \%$. Ce résultat, au niveau de la fonction publique dans son ensemble, amalgame toutefois un ensemble d'effets inhérents à la structure des postes occupés respectivement par les femmes et par les hommes. Nous reconduisons donc la décomposition pour des sous-populations de différentes catégories d'agents, A, B et C, du plus au moins élevé des niveaux hiérarchiques et des niveaux de diplôme minima pour se présenter aux concours externes. La catégorie $\mathrm{C}$ est ainsi supposée correspondre à un niveau brevet et la catégorie $\mathrm{B}$ à un niveau baccalauréat. Quant à la catégorie $\mathrm{A}$, elle équivaut au moins à un niveau bac +3 . Parmi les très nombreux salariés de la catégorie $A$, il est intéressant de distinguer ceux qui occupent des postes à responsabilités particulières, dits $\langle\mathrm{A}+\rangle^{1}$, du reste de la catégorie qui sera notée «A hors $A+»$.

Les schémas de rémunération apparaissent assez différents pour les agents B et C d'une part et A d'autre part (cf. tableau 1). Dans les catégories $\mathrm{B}$ et $\mathrm{C}$, l'inégalité des rémunérations est peu importante, comme en témoignent des indices

\footnotetext{
1. La catégorie A+ se définit comme l'ensemble des corps ou emplois fonctionnels dont un grade atteint un indice majoré strictement supérieur à 963. Viennent s'y ajouter les professions qui, ne respectant pas ce premier critère, requièrent un diplôme de doctorat ou d'ingénieur pour le recrutement. Voir Baradji et Peyrin (2011) pour une définition plus complète de la sous-catégorie A+. Les postes A+ qui représentent environ $2 \%$ dans notre base, ne sont pas directement identifiés dans les données du SIASP, la liste précise des postes classés $A+$ nous a été fournie par la DGAFP dans le cadre du contrat à l'origine de cette étude.
}

Tableau 1

Indice de Gini de la rémunération totale et contribution des facteurs âge et genre, fonction publique dans son ensemble et par catégorie

\begin{tabular}{|l|c|c|c|c|}
\hline & \multirow{2}{*}{ Indice de Gini } & Âge & Genre & Reste \\
\hline Ensemble & 0,221 & 17,2 & 8,9 & 73,9 \\
Catégorie A & 0,228 & 19,6 & 9,9, & 70,5, \\
$\quad$ Catégorie «A hors A+ » & 0,208 & 27,4 & 9,1 & 63,6 \\
Catégorie A+ & 0,245 & 30,9 & 7,6 & 61,6 \\
Catégorie B & 0,162 & 29,0 & 10,6 & 60,4 \\
Catégorie C & 0,149 & 18,3 & 11,7 & 70,0 \\
\hline
\end{tabular}

Lecture: dans la fonction publique, les inégalités de rémunération totale mesurées par l'indice de Gini prennent une valeur égale à 0,221; les contributions des facteurs âge et genre sont, respectivement, de 17,2 \% et de 8,9\%.

Champ : France entière, salariés de la fonction publique.

Source : SIASP 2010, Insee. 
de Gini de, respectivement, 0,162 et 0,149 . Ces valeurs très faibles sont la conséquence directe d'une amplitude très limitée des indices de rémunération dans ces catégories. L'inégalité des rémunérations est nettement plus prononcée au sein de la catégorie A, qu'elle soit considérée dans sa totalité (l'indice de Gini est de 0,228), ou en distinguant les postes classés « $\mathrm{A}+$ » (Gini de 0,245) des «A hors $A+»$ (Gini de 0,208).

Reste à déterminer la contribution des facteurs âge et genre dans ces différentes catégories. La répartition par catégorie des agents permet d'obtenir des sous-populations regroupant des agents plus similaires en termes de carrière que ce n'était le cas dans la population globale. Il en résulte que le facteur âge explique beaucoup mieux l'inégalité au niveau de chaque catégorie séparément que dans l'ensemble de la fonction publique. La contribution de l'âge aux inégalités est ainsi particulièrement forte pour les $\mathrm{B}(29 \%)$, les $\mathrm{A}+(30,9 \%)$ et les «A hors $\mathrm{A}+»(27,4 \%)$.

Quant à la part des inégalités attribuable au facteur genre, elle est un peu plus forte au sein de chaque catégorie qu'elle ne l'était au niveau de tout le secteur. De fait, une mesure globale de la contribution du facteur genre confond des éléments de nature diverse. L'effet obtenu dépend à la fois de la structure par catégorie des emplois et des distributions de rémunération des hommes et des femmes dans chacune d'entre elle. Or, comme les hommes se concentrent particulièrement dans les catégories $\mathrm{A}$ et $\mathrm{C}$ avec des postes $\mathrm{C}$ un peu plus nombreux que les $\mathrm{A}$, l'impact de la structure des postes atténue légèrement l'effet du facteur genre par rapport à ce qu'il est dans chaque catégorie. C'est ainsi que dans les catégories $\mathrm{A}, \mathrm{B}$ et $\mathrm{C}$, le différentiel de rémunération entre hommes et femmes est à l'origine, respectivement, de $9,9 \%, 10,6 \%$ et $11,7 \%$ de l'inégalité des rémunérations. Pour la catégorie $\mathrm{A}$, la contribution du facteur genre apparait plus importante parmi les «A hors A+» $(9,1 \%)$ que parmi les « $\mathrm{A}+»(7,6 \%)$. Ainsi, le segment le plus rémunérateur de la fonction publique, le seul où les femmes sont minoritaires, est aussi celui où la part des inégalités attribuable au facteur genre est la plus faible.

\section{Une contribution du facteur genre résultant d'écarts de rémunération qui tendent à augmenter au fur et à mesure de la carrière}

La représentation des rémunérations moyennes par âge permet de visualiser les écarts qui existent entre les hommes et les femmes agents de la fonction publique et leur évolution au fur et à mesure du déroulement de la carrière, avec les précautions nécessaires comme nous l'avons noté précédemment, sur le fait que l'âge n'est qu'une approximation imparfaite de l'avancement dans la carrière.

Dans les catégories $\mathrm{A}$ et $\mathrm{B}$, les rémunérations moyennes des hommes et des femmes sont similaires pour les plus jeunes, mais un différentiel apparaît dès 24-25 ans puis tend à augmenter avec l'âge (cf. figures I et II). Concernant la catégorie $\mathrm{C}$, l'écart apparaît dès le début de la carrière et pourrait alors résulter de disparités dans la nature des postes occupés (cf. figure III). Pour les catégories B et $\mathrm{C}$, le différentiel du niveau moyen des rémunérations tend à augmenter jusqu'à environ 40 ans, puis se stabilise. Pour la catégorie A, le décalage continue à s'accroître tout au long de la carrière.

Afin d'évaluer concrètement les conséquences financières des inégalités mesurées au tableau 1 et de leur évolution mise en lumière par les figures I à III, on examine les différences de rémunération moyennes entre les femmes et les hommes appartenant à chacune des trois catégories aux âges de 25 et 50 ans (cf. tableau 2). En 2010, les agents concernés sont ceux qui sont nés respectivement en 1985 et 1960, les premiers débutant leur carrière, les seconds en ayant réalisé la plus grande partie.

À 25 ans, la différence de rémunération entre les femmes et les hommes est assez limitée, puisque dans la catégorie $\mathrm{C}$ où cette différence est la plus importante en proportion, les femmes ne reçoivent «que » $6,3 \%$ de moins que les hommes. Dans les catégories A et B, le manque à gagner des femmes représente respectivement $3,9 \%$ et $4,2 \%$ de la rémunération moyenne des hommes.

À 50 ans, la différence de rémunération a nettement augmenté en valeur comme en proportion dans toutes les catégories. C'est dans la catégorie A que l'écart s'est le plus accru puisqu'une femme touche en moyenne 23,3\% de moins qu'un homme. En proportion, l'écart hommes-femmes a donc plus que quintuplé dans cette catégorie en vingt-cinq ans de carrière, alors qu'il n'a guère plus que doublé ou triplé dans les catégories $\mathrm{B}$ et $\mathrm{C}$, soit un manque à gagner de $14,3 \%$ et $14,5 \%$ respectivement. Cela se traduit, en fin de carrière, par un écart substantiel du montant des rémunérations 
annuelles EQTP, puisqu'à 50 ans une femme gagne en moyenne environ 3600 euros de moins qu'un homme si elle appartient à la catégorie C, contre 4900 euros dans la catégorie B, et 9900 euros si elle occupe un poste classé A.

\section{Figure I}

Rémunération totale annuelle nette EQTP par âge des agents de catégorie A

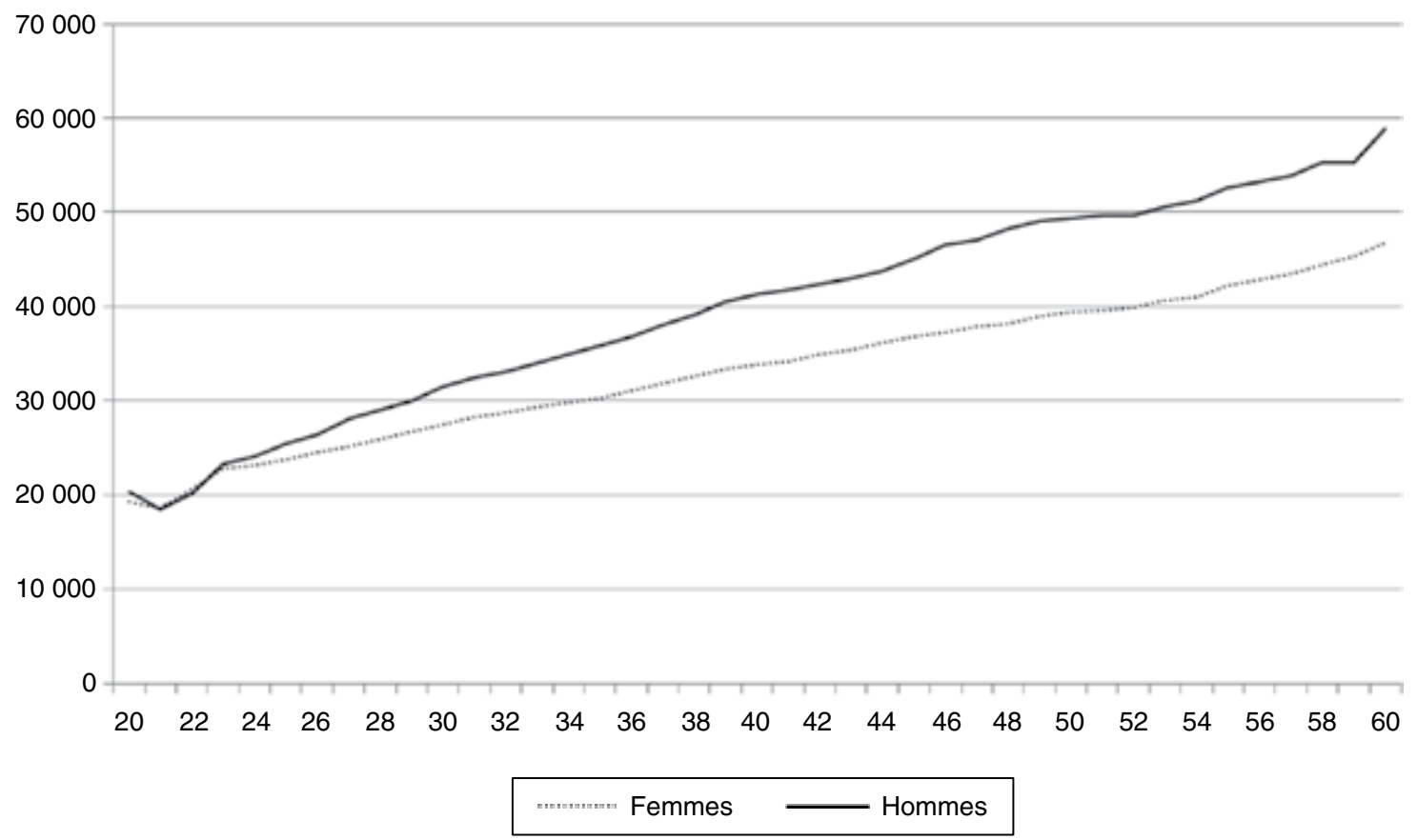

Champ : France entière, salariés de la fonction publique. Source : SIASP 2010

Figure II

Rémunération totale annuelle nette EQTP par âge des agents de catégorie B

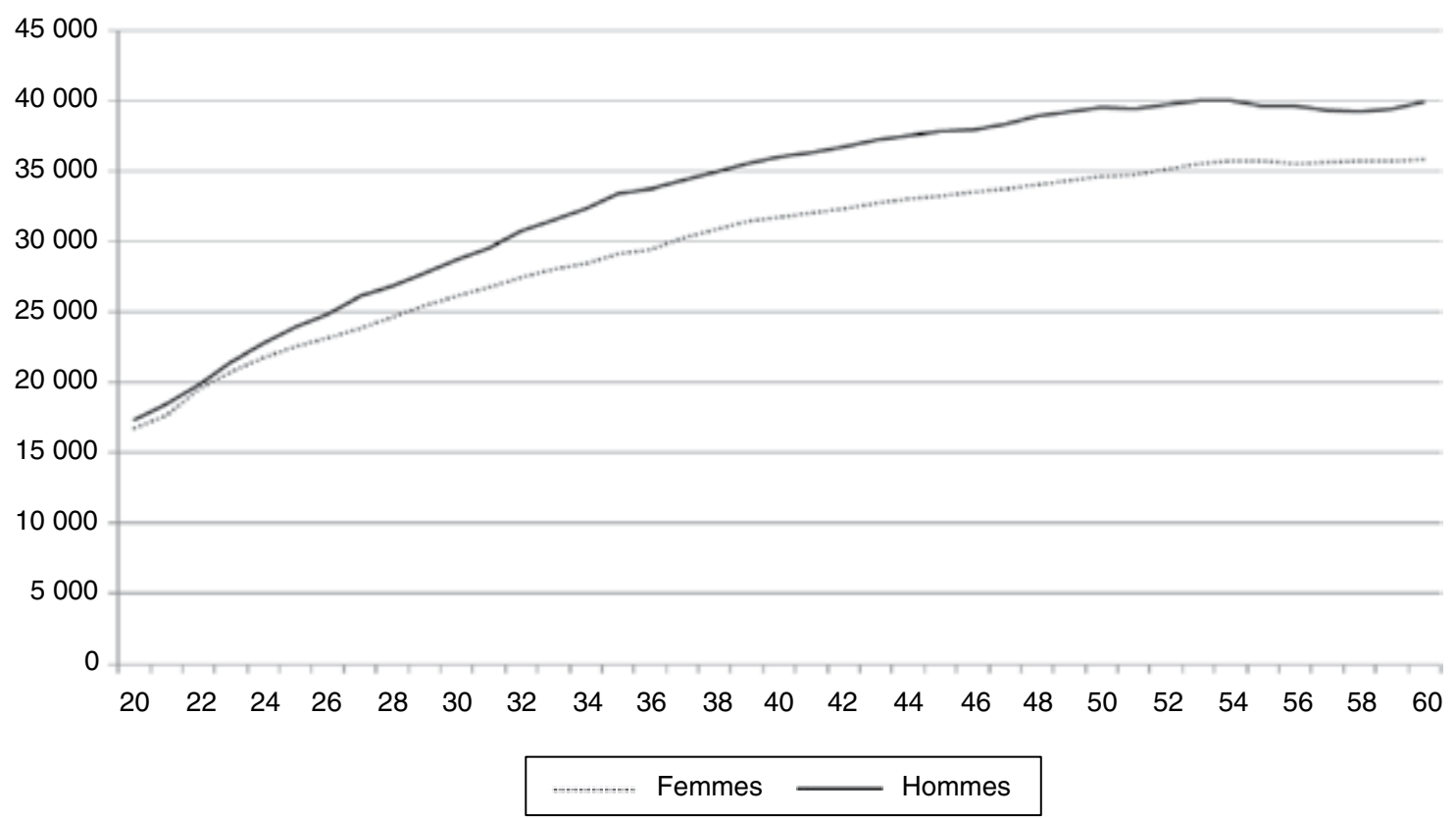

Champ : France entière, salariés de la fonction publique. Source : SIASP 2010. 
Comment comprendre que, dans chaque catégorie de la fonction publique, le fait d'être une femme puisse conduire à une rémunération EQTP en moyenne moindre que celle obtenue par un homme du même âge, et ce, en dépit d'une progression de carrière en grande partie basée sur l'ancienneté et donc équivalente pour tous les agents, y compris ceux travaillant à temps partiel ? Une première raison tient à la nature même des emplois occupés par les femmes et par les hommes. Dans une catégorie donnée, les femmes peuvent en effet occuper des emplois ouvrant des perspectives moins favorables que la moyenne de cette catégorie en termes d'indices de rémunération atteignables. Par exemple, les professeurs des écoles ou titulaires du Capes, très majoritairement des femmes, occupent des postes relativement mal rémunérés par rapport au reste de la catégorie $\mathrm{A}$.

D'autre part, les femmes ont pu accumuler moins d'ancienneté que les hommes ; si rien ne permet de penser qu'elles intègrent la fonction publique plus tardivement que les hommes, il est en revanche probable qu'elles connaissent davantage d'interruptions de carrière, comme l'indiquent de nombreuses études (voir par exemple Mairesse et Pezzoni, 2015). Sachant

Figure III

Rémunération totale annuelle nette EQTP par âge des agents de catégorie C (en euros)

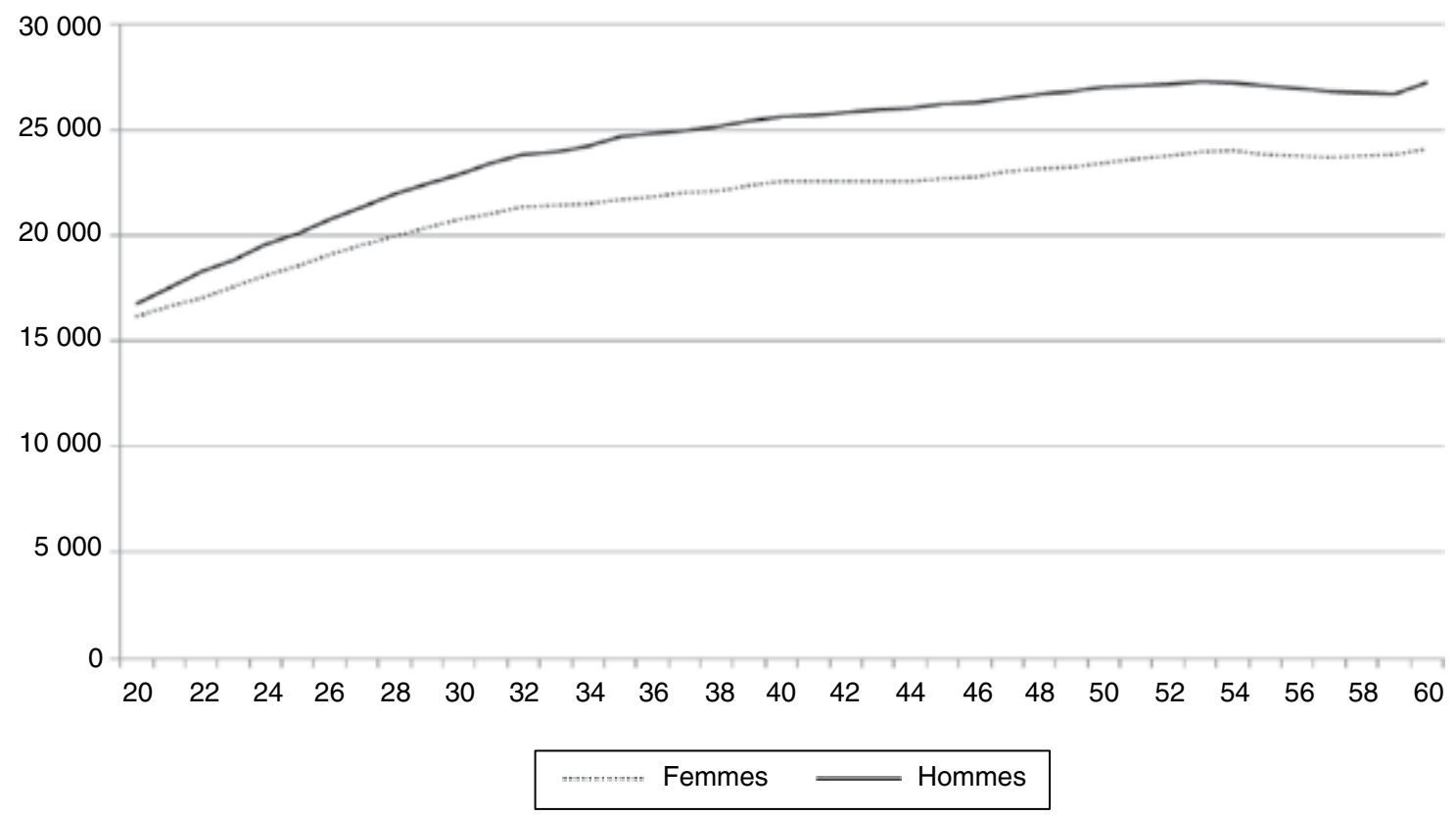

Champ : France entière, salariés de la fonction publique. Source : SIASP 2010.

Tableau 2

Différences moyennes de rémunération totale entre les hommes et les femmes aux âges de 25 et $\mathbf{5 0}$ ans par catégorie hiérarchique de la fonction publique (en euros et en proportion du salaire moyen des hommes)

\begin{tabular}{|l|c|c|c|} 
& Catégorie A & Catégorie B & Catégorie C \\
& $\begin{array}{c}\text { F-H en euros } \\
\text { (H-F)/H en \% }\end{array}$ & $\begin{array}{c}\text { F-H en euros } \\
(\mathrm{H}-\mathrm{F}) / \mathrm{H} \text { en } \%\end{array}$ & -1561 \\
& -1669 & -1431 & $-1 \mathrm{H}$ en \% \\
\hline À 25 ans & 3,9 & 4,2 & 6,3 \\
(agents nés en 1985) & -9872 & -4869 & -3589 \\
\hline À 50 ans & 23,3 & 14,3 & 14,5 \\
(agents nés en 1960) & & \\
\hline
\end{tabular}

Lecture : à 25 ans, la rémunération d'une femme occupant un poste de catégorie $A$ est en moyenne inférieure de 3,9\% à celle d'un homme de la même catégorie, soit un manque à gagner de 1669 euros.

Champ : France entière, salariés de la fonction publique.

Source : SIASP 2010, Insee. 
que de telles interruptions provoquent un retard de durée équivalente dans le déroulement de carrière, les salariées concernées obtiennent mécaniquement des rémunérations inférieures à celles des agents du même âge qui ne se sont pas arrêtés. Une dernière hypothèse serait que les femmes bénéficient relativement moins d'avancement au choix que leurs homologues masculins, ou encore qu'elles aient passé ou réussi moins de concours internes (ou simplement les aient passé plus tard ${ }^{2}$ ). Le constat d'une contribution particulièrement faible $\mathrm{du}$ genre dans la catégorie A+ (cf. tableau 1) soutient indirectement ces explications. Il est en effet logique de penser que les femmes ayant réussi les concours donnant l'accès aux emplois les plus valorisés de la fonction publique sont plus investies dans leur carrière que la moyenne des femmes du secteur, ce qui signifie notamment moins de temps partiel et moins d'interruptions de carrière. De fait, la distribution de leur rémunération reste plus proche de celle de leurs homologues masculins que dans les autres catégories.

Les données du SIASP (2010) n'apportent malheureusement pas les éléments d'information qui permettraient d'approfondir l'analyse dans ces directions. Cependant, la forme des figures I à III n'est pas incompatible avec l'hypothèse d'un effet d'interruptions de carrière liées aux maternités, car la tranche d'âge dans laquelle surviennent et se creusent les écarts correspond approximativement à cette période de la vie. Sans qu'il puisse être définitivement établi, un tel lien ne peut donc pas être exclu.

\section{Des versants concernés à des degrés très différents par les écarts de rémunération entre les hommes et les femmes}

La fonction publique ne saurait être considérée comme un tout indistinct, car elle est composée d'établissements très différents quant à leur mission et aux types d'emplois offerts. Tout d'abord, la structure par catégorie des postes est très différente d'un versant à l'autre de la fonction publique. La grande majorité des postes de la FPE, 62,2\%, sont des postes de catégorie A, contre seulement $19,2 \%$ de catégorie B et $18,6 \%$ de catégorie C. À l'inverse, 77,8\% des emplois de la FPT sont classés $\mathrm{C}$, alors que les $A$ et les $B$ ne représentent respectivement que $8,9 \%$ et $13,2 \%$ des effectifs. Quant à la $\mathrm{FPH}$, elle compte $17,9 \%$ de postes de catégorie A, $32,7 \%$ de $\mathrm{B}$ et $49,5 \%$ de $\mathrm{C}$. Contrairement à la structure des postes qui les opposent, la FPE et la FPT ont, comme nous l'avions souligné précédemment, un taux de féminisation similaire, puisque la première emploie $59,5 \%$ de femmes et la seconde $60,5 \%$. En revanche, la FPH est nettement plus féminisée avec plus de $77 \%$ de salariées.

Une analyse menée sur chaque versant séparément permet d'explorer l'impact possible de ces différences sur l'inégalité des rémunérations et la contribution du genre à cette inégalité.

L'inégalité des rémunérations totales, mesurée par l'indice de Gini, est d'une ampleur assez comparable d'un versant à l'autre, à hauteur de 0,21 environ (cf. tableau 3). En revanche la contribution du facteur genre diffère par versant. La part des inégalités attribuable au genre est assez similaire dans la FPE et la FPT, où elle s'élève respectivement à $10,5 \%$ et $11 \%$, alors qu'elle est nettement plus faible dans la FPH avec une valeur inférieure à $7 \%$. Une analyse plus détaillée, par catégorie et type d'établissement, confirme ces différences.

\section{Dans la FPH, une contribution du facteur genre uniquement dans la catégorie $A$}

Par rapport à la fonction publique dans son ensemble, la FPH présente un niveau d'inégalités plus faible dans les catégories $\mathrm{B}$ et $\mathrm{C}$ (voir les indices de Gini donnés dans les tableaux 1 et 3). La décomposition proposée au tableau 3 montre en outre que la contribution du genre à l'inégalité des rémunérations dans ces deux catégories de la FPH est particulièrement faible avec $1,1 \%$ et $2,2 \%$ respectivement pour les emplois classés $\mathrm{B}$ et $\mathrm{C}$.

Contrairement aux catégories $\mathrm{B}$ et $\mathrm{C}$, les inégalités pour la catégorie A et les sous-catégories « $\mathrm{A}+$ » et «A hors $\mathrm{A}+$ » de la FPH sont, avec des indices de Gini de, respectivement, 0,251, 0,272 et 0,257 , plus élevées que dans l'ensemble de la fonction publique (cf. tableau 3 ). Les rémunérations de ces deux sous-catégories présentent des profils très différents. En effet, la part de l'inégalité attribuable au facteur genre est de plus du double parmi les agents « A hors $\mathrm{A}+$ » de celle mesurée parmi les « $\mathrm{A}+»$ (respectivement près de $10 \%$ et à peine $4 \%$ ) ; en revanche, l'âge contribue pour plus de

2. Les femmes peuvent avoir un choix de promotion ou de mobilité plus contraint par leur vie familiale que les hommes (Franck, 1978). 
$45 \%$ à l'inégalité des rémunérations entre les « $\mathrm{A}+$ » (contre $38 \%$ pour les « $\mathrm{A}$ hors $\mathrm{A}+»)$. Ces différences s'expliquent aisément : dans la FPH, peu de postes sont classés « $\mathrm{A}+$ », puisque cela concerne uniquement les directeurs des hôpitaux et des autres établissements publics relevant de ce versant. Il en résulte apparemment une certaine homogénéité dans les profils de carrière des titulaires de ces emplois qu'il s'agisse d'hommes ou de femmes, d'où la forte contribution de l'âge et la faible contribution du genre. À l'inverse, la sous-catégorie « A hors $\mathrm{A}+$ » regroupe des emplois de natures très diverses, et notamment les postes de médecins qui comptent parmi les mieux rémunérés du versant et qui sont majoritairement occupés par des hommes contrairement aux emplois $\mathrm{du}$ reste de la sous-catégorie. La présence des médecins dans cette sous-population correspond à un effet de structure qui explique donc largement la plus forte contribution du genre dans cette catégorie. En distinguant les hôpitaux et les autres établissements publics administratifs (EPA) du versant (principalement des maisons de retraite), l'effet particulier de la présence des médecins apparaît nettement : dans la catégorie A, la part des inégalités attribuable au facteur genre est beaucoup plus importante dans les hôpitaux ( $9 \%$ ), où l'on trouve la quasi totalité des médecins, que dans les autres EPA de la FPH (5\%) (cf. tableau 4). Pour les catégories B et $\mathrm{C}$, la part des inégalités attribuable au facteur genre est aussi faible pour les salariés des hôpitaux que pour ceux des EPA.

L'analyse détaillée par catégorie, sous-catégorie et type d'établissement de la FPH fait ainsi apparaître une configuration dans laquelle le facteur genre n'explique qu'une très faible part de l'inégalité des rémunérations, avec une quasi absence de décalage entre les distributions des rémunérations des hommes et des femmes.

\section{Dans la FPE, une contribution élevée du facteur genre tout particulièrement dans les emplois des ministères}

Les inégalités mesurées au niveau de la FPE sont comparables à celles mesurées pour la fonction publique dans son ensemble (cf. tableaux 1 et 3). De même que la contribution du genre

\begin{tabular}{|c|c|c|c|c|}
\hline & \multirow{2}{*}{ Indice de Gini } & \multicolumn{3}{|c|}{ Contributions (\%) } \\
\hline & & Âge & Genre & Reste \\
\hline FPE & 0,209 & 28,6 & 10,5 & 60,9 \\
\hline A & 0,216 & 19,6 & 9,94 & 70,4 \\
\hline At & 0,256 & 30,9 & 7,6 & 61,6 \\
\hline A hors A+ & 0,190 & 27,4 & 9,1 & 63,6 \\
\hline B & 0,169 & 29,0 & 10,6 & 60,4 \\
\hline C & 0,158 & 18,3 & 11,7 & 70,0 \\
\hline FPH & 0,218 & 22,3 & 6,9 & 70,8 \\
\hline A & 0,251 & 38,1 & 8,9 & 53,0 \\
\hline At & 0,272 & 45,4 & 3,9 & 50,7 \\
\hline A hors $\mathrm{A}+$ & 0,257 & 37,8 & 9,9 & 52,4 \\
\hline B & 0,145 & 41,6 & 1,1 & 57,2 \\
\hline C & 0,129 & 26,4 & 2,2 & 71,4 \\
\hline FPT & 0,212 & 14,6 & 11,1 & 74,3 \\
\hline A & 0,216 & 22,2 & 12,7 & 65,1 \\
\hline A+ & 0,218 & 25,0 & 12,0 & 63,0 \\
\hline $\mathrm{A}$ hors $\mathrm{A}+$ & 0,188 & 17,7 & 11,1 & 71,2 \\
\hline B & 0,163 & 30,5 & 6,5 & 63,0 \\
\hline C & 0,148 & 8,7 & 14,1 & 77,3 \\
\hline
\end{tabular}

Lecture : l'indice de Gini est égal à 0,209 pour la FPE; les contributions à l'inégalité des rémunérations des facteurs âge et genre sont, respectivement, de $28,6 \%$ et de $10,5 \%$.

Champ : France entière, salariés de la fonction publique

Source : SIASP 2010, Insee. 
reste dans chaque catégorie presque identique à ce qu'elle est dans le secteur dans son ensemble, à l'exclusion de la catégorie $\mathrm{B}$ où elle est plus forte, à hauteur de 13,5 \%. Cependant, une analyse plus approfondie montre une réalité très diverse selon l'établissement considéré.

En effet, lorsque l'on restreint l'analyse aux seuls emplois situés directement dans un ministère, les indices de Gini mesurés sur les rémunérations totales diminuent légèrement dans chacune des catégories considérées (cf. annexe, tableau A1). En revanche, la part de l'inégalité attribuable au facteur genre n'en est absolument pas réduite. Bien au contraire, on constate une hausse de cette part dans les catégories $\mathrm{B}$ et $\mathrm{C}$ jusqu'à, respectivement, $15,9 \%$ et $13,2 \%$. Ces résultats semblent d'ailleurs dus à une concentration des écarts de rémunération entre les femmes et les hommes particulièrement importante dans ces emplois ministériels, ceux offerts par les autres établissements publics administratifs (EPA) de la FPE étant nettement moins concernés.

De fait, la comparaison montre à quel point les distributions des rémunérations sont différentes entre les emplois ministériels et ceux situés dans les autres EPA de la FPE. L'inégalité des rémunérations est en effet beaucoup plus élevée dans les seconds que dans les premiers, mais il est probable que cela résulte de la nature extrêmement variée des établissements publics en question qui, en 2010, regroupent aussi bien les salariés de la moitié des universités que ceux de Pôle emploi par exemple. Cela doit nous inciter à considérer avec précaution les chiffres présentés dans le tableau 5. Quoiqu'il en soit, la part des inégalités attribuable au facteur genre est très inférieure

Tableau 4

Inégalités de rémunération totale mesurées par l'indice de Gini et proportions expliquées par les facteurs âge et genre, FPH par type d'établissement

\begin{tabular}{|c|c|c|c|c|}
\hline & \multirow{2}{*}{ Indice de Gini } & \multicolumn{3}{|c|}{ Contributions (\%) } \\
\hline & & Âge & Genre & Reste \\
\hline Hôpitaux & 0,219 & 24,9 & 5,7 & 69,4 \\
\hline$A$ & 0,251 & 38,3 & 9,0 & 52,7 \\
\hline $\mathrm{B}$ & 0,144 & 42,3 & 1,2 & 56,5 \\
\hline $\mathrm{C}$ & 0,129 & 27,7 & 2,1 & 70,3 \\
\hline EPA & 0,168 & 21,9 & 4,5 & 73,6 \\
\hline A & 0,245 & 34,2 & 5,0 & 60,8 \\
\hline B & 0,156 & 37,9 & 3,0 & 59,1 \\
\hline C & 0,123 & 20,0 & 1,9 & 78,1 \\
\hline
\end{tabular}

Lecture : dans les établissements hospitaliers de la FPH, l'indice de Gini est égal à 0,219; les contributions à l'inégalité des rémunérations des facteurs âge et genre sont, respectivement, de $24,9 \%$ et de $5,7 \%$

Champ : France entière, salariés de la fonction publique hospitalière

Source : SIASP 2010, Insee.

Tableau 5

Inégalités de rémunération totale mesurées par l'indice de Gini et proportions expliquées par les facteurs âge et genre, FPT par type d'établissement

\begin{tabular}{|c|c|c|c|c|}
\hline & \multirow{2}{*}{ Indice de Gini } & Âge & Genre & Reste \\
\hline Collectivités territoriales & $\mathbf{0 , 1 9 1}$ & $\mathbf{1 2 , 1}$ & $\mathbf{6 , 6}$ & $\mathbf{8 1 , 3}$ \\
A & 0,207 & 21,5 & 11,5 & 67,0 \\
B & 0,153 & 31,3 & 4,5 & 64,3 \\
C & 0,140 & 10,8 & 10,6 & 78,7 \\
\hline EPA locaux & $\mathbf{0 , 2 2 3}$ & $\mathbf{9 , 7}$ & $\mathbf{1 8 , 8}$ & $\mathbf{7 1 , 5}$ \\
A & 0,238 & 25,0 & 14,4 & 60,6 \\
B & 0,193 & 29,3 & 11,7 & 59,1 \\
C & 0,172 & 6,5 & 25,3 & 68,2 \\
\hline
\end{tabular}

Lecture: dans les collectivités territoriales de la FPT, l'indice de Gini est égal à 0,191; les contributions à l'inégalité des rémunérations des facteurs âge et genre sont, respectivement, de $12,1 \%$ et de $6,6 \%$

Champ : France entière, salariés de la fonction publique territoriale.

Source : SIASP 2010, Insee. 
dans les EPA à ce qui a été mesuré pour les postes ministériels, puisque, par catégorie, cette part va de $2,1 \%$ pour les emplois classés $\mathrm{C}$ à $6,7 \%$ pour ceux classés A. Les écarts de rémunération entre les hommes et les femmes y sont donc assez limités. Le facteur âge ressort à l'inverse comme particulièrement explicatif de l'inégalité des rémunérations au sein des catégories A et $B$.

Dans la FPE, c'est donc dans les ministères que le différentiel de rémunération entre les hommes et les femmes est le plus important, et ce résultat est maintenu une fois pris en compte les principaux effets de structure, puisque la contribution du facteur genre est aussi forte au sein de chaque catégorie qu'au niveau du versant dans son ensemble. Cependant, il n'est pas évident qu'il en soit de même dans tous les ministères, et une analyse détaillée par ministère montre en effet que tel n'est pas le cas (cf. figure IV et annexe, tableau A2).

Pour dix des quinze ministères recensés dans le tableau A2, la contribution du genre toutes catégories confondues est supérieure à $10 \%$, et pour quatre d'entre eux (les Affaires étrangères et européennes, l'Intérieur, outre-mer et collectivités territoriales, l'Immigration, intégration, identité nationale et développement solidaire, ainsi que les Services du Premier ministre), elle est supérieure à $15 \%$, alors qu'elle n'est inférieure à $5 \%$ que pour le seul ministère de la Culture et de la communication.
Les disparités entre ministères paraissent donc très fortes, et il est intéressant de comparer les structures des rémunérations par âge et sexe de deux ministères, Culture et Intérieur, totalement opposés en termes d'inégalités de genre (cf. figure V) : au ministère de la Culture, les rémunérations totales des hommes et des femmes restent proches à tous âges, et il en résulte une part des inégalités attribuable au genre extrêmement faible $(3,5 \%$, cf. annexe, tableau A2). Il ne semble donc pas y avoir de réel décalage entre les carrières des hommes et des femmes au sein de ce ministère. À l'inverse, dans le cas du ministère de l'Intérieur, les salaires des femmes et des hommes divergent nettement au-delà de 30 ans. Il en résulte que $17,1 \%$ des inégalités de rémunération sont expliquée par le genre dans ce ministère.

Il faut cependant se méfier, pour ces deux ministères, comme pour les autres, des chiffres toutes catégories confondues, car ils pourraient être affectés de biais structurels, et il faut procéder à une analyse par catégorie pour s'en abstraire. Ainsi au ministère de l'Intérieur ou, dans une moindre mesure, dans les Services du Premier ministre, où la contribution du genre aux inégalités semblait particulièrement importante, elle est beaucoup plus limitée par catégorie (cf. annexe, tableau A2). En revanche, les écarts de rémunération restent élevés dans le cas des Affaires étrangères et européennes ou de l'Immigration.

Figure IV

Contribution du facteur genre à l'inégalité des rémunérations dans les emplois ministériels

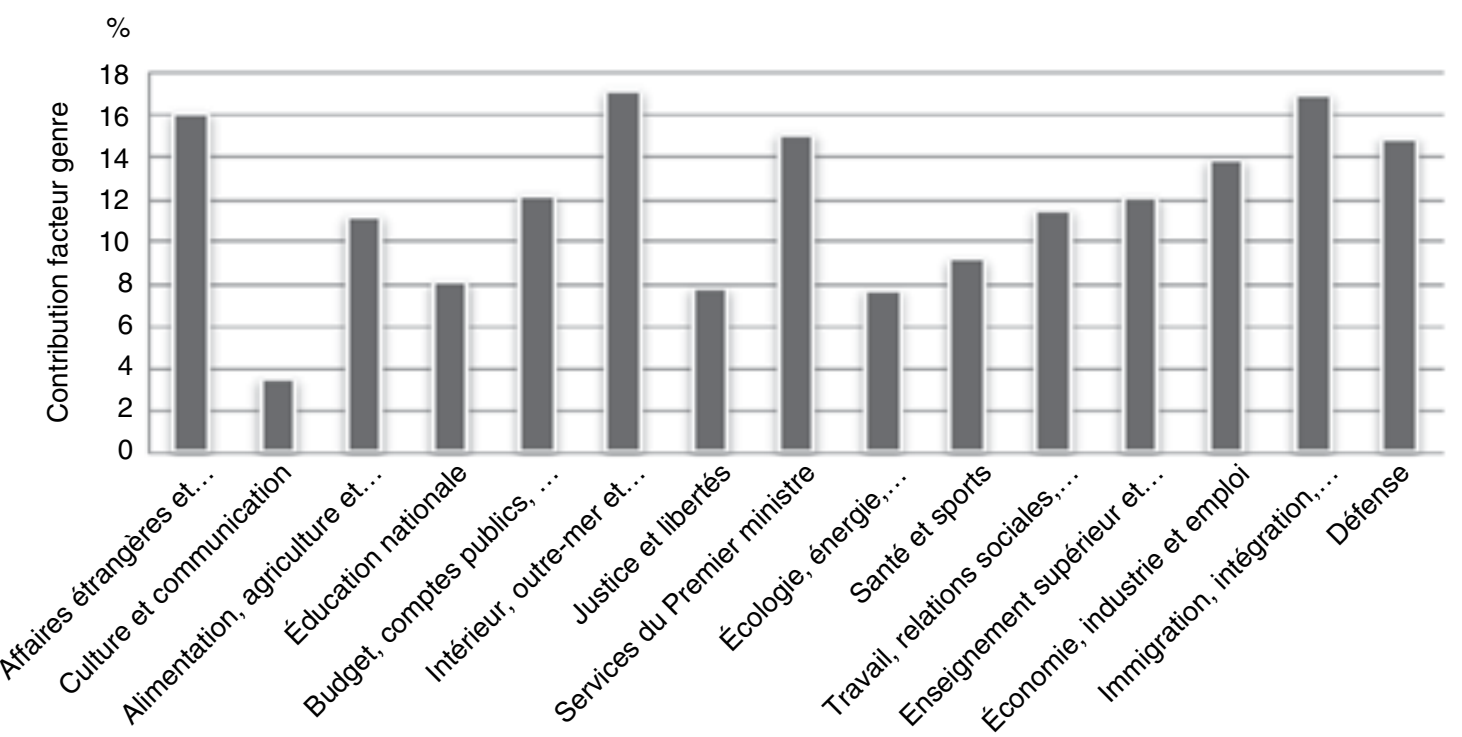

Champ : France entière, salariés de la fonction publique. Source : SIASP 2010. 
Figure V

Rémunération totale annuelle nette EQTP par âge et sexe

A. Ministère de la Culture et de la communication

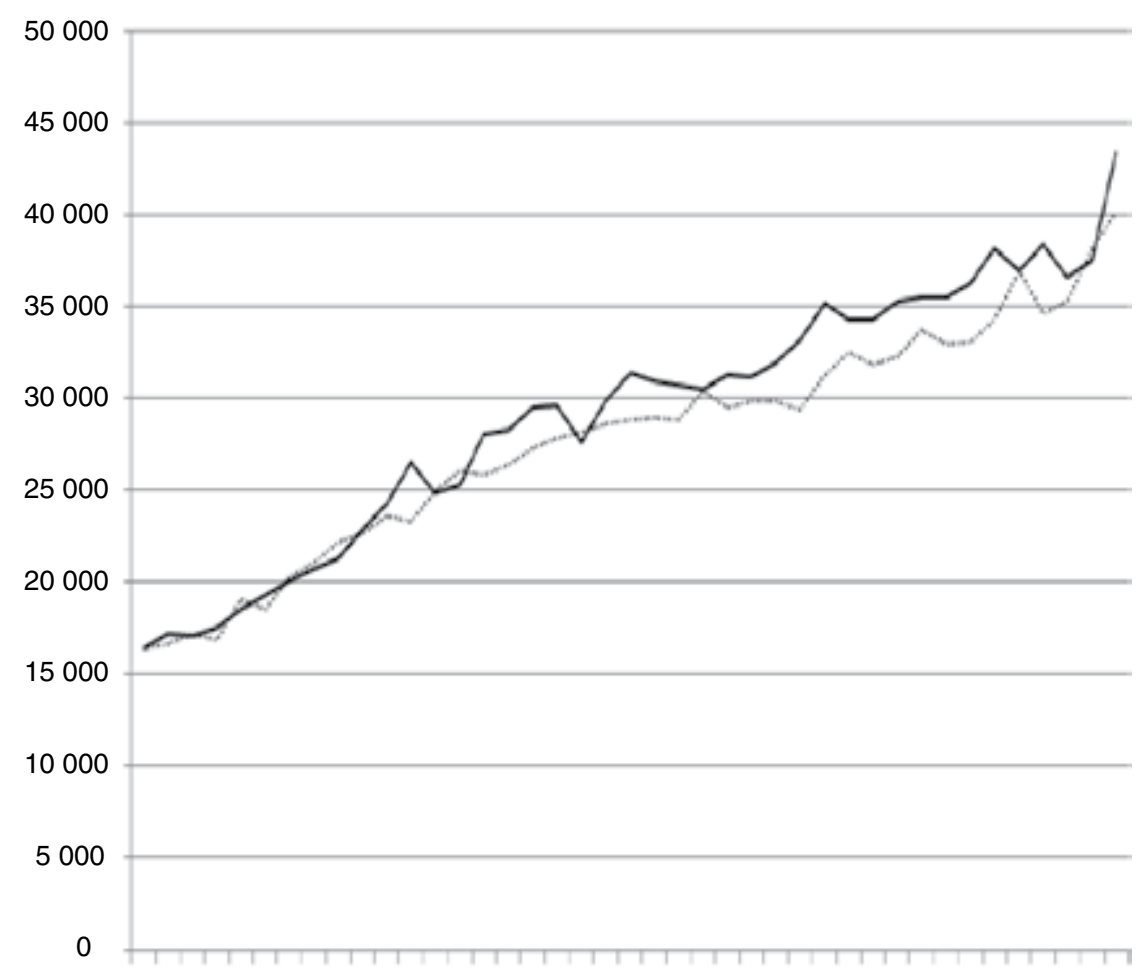

$\begin{array}{lllllllllllllllllllll}20 & 22 & 24 & 26 & 28 & 30 & 32 & 34 & 36 & 38 & 40 & 42 & 44 & 46 & 48 & 50 & 52 & 54 & 56 & 58 & 60\end{array}$ Femmes $\longrightarrow$ Hommes

B. Ministère de l'Intérieur, de l'outre-mer et des collectivités territoriales

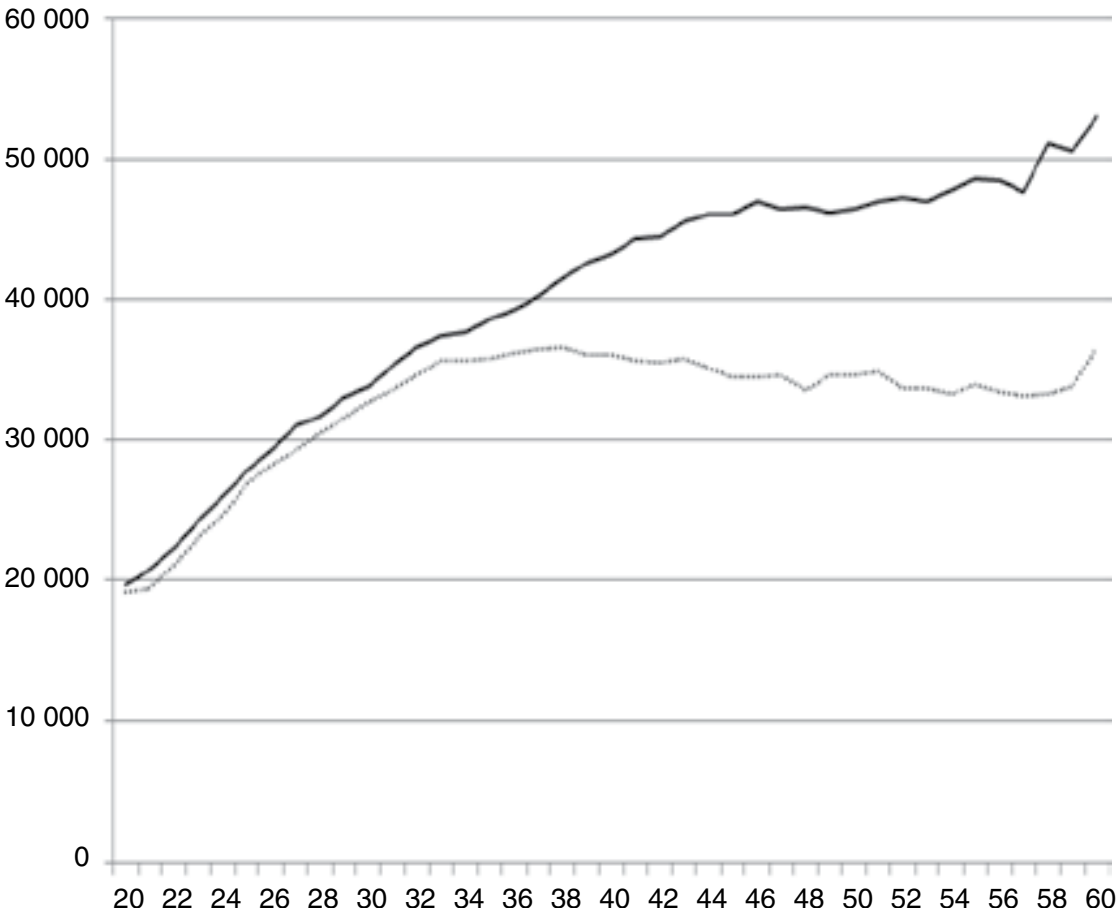

$$
\text { Femmes Hommes }
$$

Champ : France entière, salariés de la fonction publique d'État. Source : SIASP 2010 


\section{Dans la FPT, de fortes contributions du facteur genre dans les catégories $A$ et $\mathrm{C}$, et plus dans les autres établissements publics administratifs locaux que dans les collectivités territoriales}

En termes de niveau d'inégalité, la FPT est globalement proche de la fonction publique dans son ensemble (cf. tableaux 1 et 3), mais la décomposition de l'inégalité est extrêmement spécifique au versant. La catégorie B mise à part, toutes les catégories et sous-catégories de la FPT présentent une part d'inégalité attribuable au genre particulièrement importante (cf. tableau 3). Ainsi, les contributions du facteur genre à l'inégalité des rémunérations dans la catégorie A et les sous-catégories A+ et " $\mathrm{A}$ hors $\mathrm{A}+$ ", sont respectivement égales à $12,7 \%, 11,1 \%$ et $12 \%$. Mais le plus spécifique à la FPT s'observe pour la catégorie C. La part de l'inégalité attribuée au facteur genre y est en effet particulièrement élevée, à hauteur de $14,1 \%$. En parallèle, l'âge contribue très peu à l'inégalité des rémunérations entre les agents de cette catégorie $(8,7 \%)$.

L'inégalité est également plus marquée dans les EPA locaux que dans les collectivités territoriales quelle que soit la catégorie considérée (cf. tableau 5). Mais surtout et avant tout, c'est l'ampleur de la contribution du genre à l'inégalité qui différencie ces deux pans de la FPT.

Bien que cette contribution soit notable aussi pour les collectivités territoriales, notamment pour les catégories $\mathrm{A}$ et $\mathrm{C}$ pour lesquelles la proportion attribuable au facteur genre atteint respectivement $11,5 \%$ et $10,6 \%$, les niveaux observés y restent très inférieurs à ce qui est constaté dans les EPA locaux. Pour les emplois qui relèvent de ces derniers, les contributions mesurées dans le cadre de nos décompositions atteignent des niveaux plus élevés que dans n'importe quel autre secteur de la fonction publique, avec des pourcentages de $14,4 \%, 11,7 \%$ et $25,3 \%$ respectivement pour les rémunérations totales des catégories $\mathrm{A}, \mathrm{B}$ et $\mathrm{C}$.

La combinaison de ces proportions extrêmement importantes, notamment pour la catégorie $\mathrm{C}$, et d'un haut niveau de l'inégalité des rémunérations ne peut qu'aboutir à des manques à gagner considérables pour les femmes salariées de ces EPA locaux par rapport aux hommes qui y sont également employés.

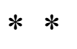

$*$

L'analyse de la contribution d'un «facteur genre » à l'inégalité des rémunérations dans une fonction publique subdivisée par type d'établissement et par catégorie a mis en lumière dans la plupart des cas un décalage entre les distributions des rémunérations des hommes et des femmes, en défaveur de ces dernières. La comparaison des contributions ainsi mesurées a cependant souligné d'importantes disparités de situation. La fonction publique hospitalière ne connaît en effet que très peu d'écarts de rémunération hommes-femmes, alors que la fonction publique d'État et la fonction publique territoriale sont beaucoup plus concernées. Au sein de ces deux derniers versants, notre étude indique aussi des différences importantes selon le type d'établissement, avec une contribution du facteur genre à l'inégalité plus marquée pour les ministères dans la FPE, et pour les autres établissements publics locaux dans la FPT.

Les résultats de notre étude ne doivent être interprétés que pour ce qu'ils sont : une photographie de la structure de l'inégalité des rémunérations dans la fonction publique en 2010 qui ne présume pas de l'origine des écarts observés entre les hommes et les femmes. En effet, les données disponibles ne nous permettent pas d'aller au-delà sur ce point. Outre les difficultés à évaluer la longueur réelle de la carrière des agents que nous avons déjà mis en lumière, d'autres informations importantes font défaut. Connaître la nature du concours d'entrée dans la fonction publique de chaque salarié aurait notamment permis de comparer les carrières des hommes et des femmes entrés à un niveau identique dans la fonction publique. Cela permettrait de comprendre ce que les décalages de rémunération doivent à une moindre performance des femmes en termes d'évolution de carrière et ce qui résulte de la concentration des femmes dans des postes ouvrant des perspectives moins favorables que la moyenne de leur catégorie et/ou de leur versant.

Rappelons aussi que la stabilité de l'emploi dont bénéficient les titulaires de la fonction publique fait que le différentiel de rémunération observé en fin de carrière reflète autant les décisions prises dans la période récente que dix ou vingt ans auparavant, qu'il s'agisse des décisions des agents eux-mêmes ou de celles prises à leur égard. En effet, les processus d'avancement impliquent une inertie dans 
les carrières telle que les écarts constatés ne sont donc pas contradictoires avec la volonté actuelle de respecter l'égalité professionnelle entre les hommes et les femmes dans la fonction publique. Seul un suivi à moyen et à long terme de la part des inégalités expliquée par le genre donnerait les éléments nécessaires pour juger de l'impact réel d'une évolution des pratiques. À court terme, connaissant les âges auxquels les rémunérations des hommes et des femmes divergent, une étude pourrait se concentrer sur les raisons et les choix qui expliquent la survenance de ces écarts, afin de poursuivre les efforts qui ont été entrepris pour garantir l'égalité de tous les agents. En mettant en lumière les différences qui existent entre les versants, notre étude montre finalement l'importance pour des travaux futurs portant sur ces questions de ne pas considérer la fonction publique comme un ensemble homogène.

\section{BIBLIOGRAPHIE}

Baradji E. et Payrin A. (2011), « L'encadrement supérieur dans les ministères : première caractérisation exhaustive des agents appartenant au corps A+ en 2007 », Dossier 2.1 du Rapport annuel sur l'état de la fonction publique 2010-2011, DGAFP, La Documentation française.

Bunel M., Chantreuil F., Gavrel F., Guironnet J.-P. et Lebon I. (2014), «Décomposition des inégalités liées au genre au sein de la fonction publique », Rapport réalisé pour le compte de la Direction Générale de l'Administration et de la Fonction Publique (DGAFP) et du Défenseur des Droits (DDD). Téléchargeable sur http:// crem.univ-rennes1.fr/Documents/Docs_eq_epcs/ Decomposition_des_inegalites_liees_au_genre_ au_sein_de_la_fonction_publique.pdf.

Chantreuil F. et Lebon I. (2015), «Gender contribution to income inequality », Economics Letters, vol. 133, pp. 27-30.

Chantreuil F. et Trannoy A. (2011), « Inequality Decomposition Values », Annals of Economics and Statistics, $\mathrm{n}^{\circ} 101-102$, pp. 13-36.

Chantreuil F. et Trannoy A. (2013), « Inequality Decomposition Values: the Trade-off between Marginality and Efficiency ", The Journal of Economic Inequality, vol. 11, n ${ }^{\circ}$, pp. 83-98.

DGAFP (2012), Rapport sur l'état de la fonction publique. Politiques et pratiques de ressources humaines. Faits et chiffres, La Documentation française.
Eidelman A. (2012), « La valeur de Shapley Comment individualiser le résultat d'un groupe », Insee, Direction des statistiques démographiques et sociales, Document de travail ${ }^{\circ} \mathrm{F} 1202$.

Frank R.H. (1978), « Why Women Earn Less: The Theory and Estimation of Differential Overqualification », American Economic Review, vol. 68, $\mathrm{n}^{\circ} 3$, pp. 360-373.

Guégot F. (2011), L'égalité professionnelle hommes-femmes dans la fonction publique, La Documentation française.

Koubi M., Mussard S., Seyte, F. et Terraza M. (2005), «Évolution des inégalités salariales en France entre 1976 et 2000 : une étude par la décomposition de l'indicateur de Gini », Économie et Statistique, $\mathrm{n}^{\circ}$ 169-170-171, pp. 139-169.

Mairesse J. et Pezzoni, M. (2015), « Does Gender Affect Scientific Productivity? A critical review of the empirical evidence and a panel data econometric analysis for French physicists ", Revue Economique, vol. $66, \mathrm{n}^{\circ} 1$, pp. $65-113$.

Shapley L. S. (1953), «A value for n-person game », dans H.W. Kuhn and A.W. Tucker (Eds), Contributions to the Theory of Games, vol. 2, Annals of Mathematics Studies, pp. 307-317

SIASP (2010), « Système d'Information sur les Agents des Services Publics, Guide d'utilisation du fichier POSTE », Insee, Direction des statistiques démographiques et sociales. 
Tableau A1

Inégalités de rémunération totale mesurées par l'indice de Gini et proportions expliquées par les facteurs âge et genre, FPE par type d'établissement et catégorie hiérarchique

\begin{tabular}{|l|c|c|c|c|}
\hline & \multirow{2}{*}{ Indice de Gini } & Âge & Genre & Reste \\
\hline Ministères & 0,196 & 20,7 & 11,8 & 67,5 \\
Ministères / A & 0,212 & 27,3 & 10,5 & 62,2 \\
Ministères / B & 0,134 & 16,0 & 15,9 & 68,2 \\
Ministères / C & 0,135 & 12,7 & 13,2 & 74,2 \\
\hline EPA & 0,268 & 32,5 & 10,7 & 56,8 \\
EPA / A & 0,220 & 41,9 & 6,7 & 51,4 \\
EPA / B & 0,218 & 44,6 & 4,1 & 51,4 \\
EPA / C & 0,175 & 19,0 & 2,1 & 78,9 \\
\hline
\end{tabular}

Champ : France entière, salariés de la fonction publique d'État.

Source : SIASP 2010.

Tableau A2

Inégalité totale des rémunérations mesurées par l'indice de Gini et proportion expliquée par le facteur genre dans les emplois des différents ministères

\begin{tabular}{|c|c|c|c|c|c|c|}
\hline & Gini & Genre (\%) & Gini & Genre(\%) & Gini & Genre(\%) \\
\hline Ministère : & \multicolumn{2}{|c|}{ Affaires étrangères et européennes } & \multicolumn{2}{|c|}{ Culture et communication } & \multicolumn{2}{|c|}{ Alimentation, agriculture et pêche } \\
\hline Ensemble & 0,264 & 16,1 & 0,226 & 3,5 & 0,234 & 11,2 \\
\hline A & 0,239 & 12,6 & 0,224 & 4,1 & 0,253 & 6,9 \\
\hline$A+$ & 0,203 & 11,7 & 0,265 & 9,0 & 0,269 & 5,1 \\
\hline B & 0,098 & 9,2 & 0,129 & 2,8 & 0,161 & 6,9 \\
\hline C & 0,188 & 9,6 & 0,132 & 4,2 & 0,146 & 7,8 \\
\hline Ministère : & \multicolumn{2}{|c|}{ Éducation nationale } & \multicolumn{2}{|c|}{$\begin{array}{l}\text { Budget, comptes publics, fonction } \\
\text { publique et réforme de l'Etat }\end{array}$} & \multicolumn{2}{|c|}{$\begin{array}{c}\text { Intérieur, outre-mer et collectivités } \\
\text { territoriales }\end{array}$} \\
\hline Ensemble & 0,171 & 8,1 & 0,210 & 12,2 & 0,179 & 17,1 \\
\hline$A$ & 0,157 & 7,6 & 0,212 & 6,3 & 0,180 & 8,0 \\
\hline$A+$ & 0,129 & 8,3 & 0,236 & 7,2 & 0,143 & 4,4 \\
\hline$B$ & 0,179 & 1,6 & 0,102 & 5,3 & 0,105 & 6,4 \\
\hline C & 0,117 & 1,4 & 0,101 & 5,8 & 0,122 & 3,9 \\
\hline Ministère : & \multicolumn{2}{|c|}{ Justice et libertés } & \multicolumn{2}{|c|}{ Services du Premier ministre } & \multicolumn{2}{|c|}{$\begin{array}{l}\text { Écologie, énergie, développement } \\
\text { durable et mer }\end{array}$} \\
\hline Ensemble & 0,239 & 7,8 & 0,361 & 15,1 & 0,286 & 7,7 \\
\hline A & 0,239 & 2,8 & 0,321 & 8,0 & 0,269 & 4,9 \\
\hline$A+$ & 0,095 & 5,8 & 0,255 & 6,3 & 0,333 & 5,4 \\
\hline B & 0,121 & 4,7 & 0,134 & 8,3 & 0,175 & 6,5 \\
\hline $\mathrm{C}$ & 0,127 & 22,0 & 0,139 & 8,4 & 0,138 & 5,5 \\
\hline Ministère : & \multicolumn{2}{|c|}{ Santé et sports } & \multicolumn{2}{|c|}{$\begin{array}{c}\text { Travail, relations sociales, famille, } \\
\text { solidarité et ville }\end{array}$} & \multicolumn{2}{|c|}{$\begin{array}{l}\text { Enseignement supérieur et } \\
\text { recherche }\end{array}$} \\
\hline Ensemble & 0,242 & 9,1 & 0,229 & 11,5 & 0,237 & 12,1 \\
\hline$A$ & 0,211 & 4,9 & 0,234 & 7,2 & 0,197 & 5,9 \\
\hline$A+$ & 0,227 & 7,5 & 0,276 & 10,4 & 0,187 & 5,9 \\
\hline B & 0,130 & 8,3 & 0,109 & 3,1 & 0,115 & 1,8 \\
\hline $\mathrm{C}$ & 0,165 & 11,2 & 0,121 & 4,8 & 0,125 & 8,0 \\
\hline Ministère : & \multicolumn{2}{|c|}{ Économie, industrie et emploi } & \multicolumn{2}{|c|}{$\begin{array}{l}\text { Immigration, intégration, identité } \\
\text { nationale et développement solidaire }\end{array}$} & \multicolumn{2}{|c|}{ Défense } \\
\hline Ensemble & 0,256 & 13,8 & 0,282 & 16,9 & 0,188 & 14,9 \\
\hline$A$ & 0,245 & 5,3 & 0,232 & 24,4 & 0,208 & 4,1 \\
\hline$A+$ & 0,216 & 6,0 & 0,172 & 6,1 & 0,394 & 7,2 \\
\hline B & 0,103 & 4,4 & 0,125 & 14,8 & 0,120 & 8,5 \\
\hline C & 0,15 & 12,7 & 0,147 & 11,5 & 0,144 & 18,2 \\
\hline
\end{tabular}

Champ : France entière, salariés de la fonction publique d'État. Source : SIASP 2010. 